\title{
Efficacy of inhaled HYdrogen on neurological outcome following BRain Ischemia During post-cardiac arrest care (HYBRID II trial): study protocol for a randomized controlled trial
}

\author{
Tomoyoshi Tamura ${ }^{1,2^{*}}$ D, Kei Hayashida ${ }^{1,2}$, Motoaki Sano ${ }^{2,3}$, Shuko Onuki ${ }^{2}$ and Masaru Suzuki ${ }^{1,2}$
}

\begin{abstract}
Background: Hydrogen gas inhalation (HI) improved survival and neurological outcomes in an animal model of post-cardiac arrest syndrome (PCAS). The feasibility and safety of $\mathrm{HI}$ for patients with PCAS was confirmed in a pilot study. The objective of this study is to evaluate the efficacy of HI for patients with PCAS.

Methods/design: The efficacy of inhaled HYdrogen on neurological outcome following BRain Ischemia During post-cardiac arrest care (HYBRID II) trial is an investigator-initiated, randomized, double-blind, placebo-controlled trial designed to enroll 360 adult comatose (Glasgow Coma Scale score $<8$ ) patients who will be resuscitated following an out-of-hospital cardiac arrest of a presumed cardiac cause. The patients will be randomized (1:1) to either the HI or control group. Patients in the HI group will inhale $2 \%$ hydrogen with $24 \%$ to $50 \%$ oxygen, and those in the control group will inhale $24 \%$ to $50 \%$ oxygen for $18 \mathrm{~h}$ after admission via mechanical ventilation. Multidisciplinary post-arrest care, including targeted temperature management (TTM) between $33^{\circ} \mathrm{C}$ and $36^{\circ} \mathrm{C}$, will be provided in accordance with the latest guidelines. The primary outcome of interest is the 90-day neurological outcome, as evaluated using the Cerebral Performance Categories scale (CPC). The secondary outcomes of interest are the 90-day survival rate and other neurological outcomes. This study will provide $80 \%$ power to detect a $15 \%$ change in the proportion of patients with good neurological outcomes (CPCs of 1 and 2), from 50\% to 65\%, with an overall significance level of 0.05 .
\end{abstract}

Discussion: The first multicenter randomized trial is underway to confirm the efficacy of $\mathrm{HI}$ on neurological outcomes in comatose out-of-hospital cardiac arrest survivors. Our study has the potential to address HI as an appealing and innovative therapeutic strategy for PCAS in combination with TTM.

Trials registration: University Hospital Medical Information Network (UMIN), 000019820. Registered on 17 November 2015

Keywords: Out-of-hospital cardiac arrest, post-cardiac arrest syndrome, hydrogen gas inhalation

\footnotetext{
* Correspondence: tamura8@keio.jp

1 Department of Emergency and Critical Care Medicine, Keio University

School of Medicine, 35 Shinanomachi, Shinkuku-ku, Tokyo 160-8582, Japan

${ }^{2}$ The Center for Molecular Hydrogen Medicine, Keio University, 2-15-45 Mita

Minato-ku, Tokyo 108-8345, Japan

Full list of author information is available at the end of the article
} 


\section{Background}

Anoxic neurological injury is a significant source of morbidity and mortality in cardiac arrest (CA) survivors [1]. Currently, targeted temperature management (TTM) is the only treatment that has both laboratory and clinical supportive data and is used to improve outcomes in patients with post-cardiac arrest syndrome (PCAS) [2, 3]. Although the optimum protocol for TTM has yet to be established, maintaining the body temperature between $32{ }^{\circ} \mathrm{C}$ and $36{ }^{\circ} \mathrm{C}$, rather than not treating the fever, is now implemented as the gold standard for improving outcomes in patients with PCAS [4, 5]. Several drugs that block toxic metabolite production have shown promise in mitigating anoxic neurological injuries in animal PCAS models. However, these drugs have not been demonstrated to improve outcomes in clinical trials [6, 7]. Thus, a medical breakthrough is warranted in post-CA care, and we are in pursuit of a novel and simple therapeutic approach.

The unique antioxidative and antiapoptotic properties, as well as the potential therapeutic applications, of molecular hydrogen $\left(\mathrm{H}_{2}\right)$ were first reported in 2007 [8]. Since then, the efficacy of $\mathrm{H}_{2}$ has been extensively studied in various animal models and preliminary clinical studies. In addition to its radical-scavenging effect, $\mathrm{H}_{2}$ has been reported to regulate gene expression and various signal transduction pathways by modifying the free radical chain reaction-dependent generation of oxidized phospholipid mediators [9]. Although the specific mechanisms underlying the pleiotropic effects demonstrated by $\mathrm{H}_{2}$ in various animal models have not been fully elucidated, the clinical translation of $\mathrm{H}_{2}$ is now attracting positive attention [10]. Improved survival rates, better neurological outcomes, and attenuated histological damage have been reported with hydrogen gas inhalation (HI) in a PCAS rat model. The salutary effect of $\mathrm{H}_{2}$ was comparable to that of TTM [11, 12]. With the unique features of $\mathrm{H}_{2}$, specifically its pleiotropic effects, high permeability, convenient administration, and lack of obvious adverse effects, HI has the potential to improve survival and neurological outcomes in patients with PCAS. Recently, we reported that $\mathrm{HI}$ is a feasible and safe approach for selected comatose postCA patients [13]. Therefore, in this trial, we will evaluate whether $\mathrm{HI}$ is effective, compared with conventional oxygen inhalation, in improving 90-day neurological outcomes in comatose patients resuscitated after out-ofhospital cardiac arrest (OHCA) of a presumed cardiac cause.

\section{Methods/design}

\section{Study design}

The Efficacy of inhaled HYdrogen on neurological outcome following BRain Ischemia During post-cardiac arrest care (HYBRID II) trial is an investigator-initiated, randomized, placebo-controlled, double-blind multicenter superiority trial (RCT) with two parallel groups. Patients will be allocated 1:1 to either the intervention group or the control group. This RCT will be conducted at approximately 15 institutions in Japan. The goal of this trial is to evaluate the efficacy of HI on neurological outcomes in comatose patients who are resuscitated following OHCA of a presumed cardiac cause. This trial is registered with the University Hospital Medical Information Network (UMIN) clinical trials registry (UMIN000019820). A completed Standard Protocol items: Recommendations for Interventional Trials (SPIRIT) Checklist is available in Additional file 1.

\section{Inclusion criteria}

The inclusion criteria are as follows: patients aged between 20 and 80 years; OHCA of a presumed cardiac cause with return of spontaneous circulation (ROSC) in a prehospital setting or in the emergency room (ER); unconscious (Glasgow Coma Scale [GCS] score $<8$ ) after ROSC; systolic blood pressure $(\geq 80 \mathrm{mmHg})$ with or without fluid loading, vasopressors, and/or inotropes; written informed consent obtained from the patient's next of kin; and $<6 \mathrm{~h}$ lapsed after ROSC before $\mathrm{HI}$ initiation.

\section{Exclusion criteria}

The exclusion criteria are as follows: known prearrest Cerebral Performance Categories scale (CPC) 3 or 4, known limitations in therapy and "do not resuscitate" order, association with trauma, intracranial bleeding, acute stroke, acute aortic dissection, malignancy in terminal stage, pregnancy, acute intoxication, oxygen $\left(\mathrm{O}_{2}\right)$ saturation $<94 \%$ with $50 \% \mathrm{O}_{2}$ inhalation and adequate positive end-expiratory pressure (PEEP), cardiopulmonary bypass use, and determined to be inappropriate for the study by the study investigators.

\section{Enrollment and withdrawal}

The study participants are unconscious and hence are unable to provide consent themselves. Therefore, before a subject's participation in the trial and after a full explanation of the study's purpose and procedures, written informed consent will be obtained by site investigators from the patient's next of kin present at the hospital. The participants will be asked whether they wish to continue participating in the study after they regain competent consciousness. The patient will be withdrawn from the study if the patient or the patient's next of kin withdraws consent. All data obtained from that patient will be discarded. Patients will be enrolled by investigators at each hospital. 


\section{Randomization, blinding, and allocation}

Owing to the limitations in transporting large gas cylinders in a timely manner and storing them in limited spaces at facilities, pairwise transportation and randomization will be performed. Thus, cylinders will be sequentially numbered from 1 to 360, and each of the 180 pairs (e.g., 1 and 2, 3 and $4, \ldots$ ) will be transported simultaneously from a storage center to a facility upon request. One of each pair of gas cylinders will contain hydrogen gas, and the other will contain a control gas. Because two cylinders will be used per patient, each pairwise shipment will contain four cylinders. Which of the gas cylinders of each pair contains hydrogen or control gas will be determined by selecting a random binary number generated by the Bernoulli distribution (probability 0.5 ) using a computer, and this information will be entered into an allocation table. The allocation table will be kept secret by the assignment manager until the time of the key opening. Before a pair of gas cylinders is transported from a storage center, the gas cylinders will be covered by cylindrical covers so that the contents of the cylinders (hydrogen or control gas) cannot be recognized. Every set of two patients at each center will be randomly allocated 1:1 to the hydrogen and control groups while maintaining blindedness.

\section{Study intervention}

The intervention is the inhalation of the blinded trial gas, which will be initiated upon admission to the intensive care unit (ICU) and will be continued for $18 \mathrm{~h}$, along with TTM (Fig. 1). The $\mathrm{H}_{2}$ group will be ventilated with $2 \% \mathrm{H}_{2}$ and titrated $\mathrm{O}_{2}$, whereas the control group will be ventilated with conventional titrated $\mathrm{O}_{2}$ through the gas inhalation system, as described below.

\section{Gas inhalation system}

The inhalation of the trial gas will be conducted using a previously described system [13]. The study gas will be supplied from a cylinder with constant flow and will be mixed with oxygen from the mechanical ventilator (SERVO-s ${ }^{\oplus}$; MAQUET Critical Care AB, Solna, Sweden) at the inspiratory duct. The cylinder is filled with $4 \% \mathrm{H}_{2}$ and $96 \%$ nitrogen for the HI group and with 100\% nitrogen for the control group (Fig. 2). Thus, the maximum $\mathrm{O}_{2}$ concentration is limited to $50 \%$ with our current $\mathrm{HI}$ system because prefilled $4 \% \mathrm{H}_{2}$ in $\mathrm{N}_{2}$ is mixed with oxygen to obtain $2 \% \mathrm{H}_{2}$ containing $\mathrm{O}_{2}$. The optimum combination values of tidal volume, respiratory rate, fraction of inspired oxygen, and trial gas flow rate, which are common for both groups, have been predetermined to obtain the above-mentioned gas composition. In our HI system, there is a discrepancy in both volume and composition between the gas emitted from the ventilator and the gas inhaled by the patient. To overcome this phenomenon, we need to deactivate the alarm on the ventilator, which works as a fail-safe under ordinary circumstances. Alternatively, we will install extra devices to monitor the tidal volume and $\mathrm{O}_{2}$ concentration throughout the intervention to ensure safety.

\section{Mechanical ventilation}

During the intervention, patients will be mechanically ventilated with mandatory volume-control ventilation according to the predefined combination. The oxidation target is peripheral oxygen saturation $\left(\mathrm{SpO}_{2}\right) \geq 94 \%$ or partial pressure of oxygen in arterial blood between $85 \mathrm{mmHg}$ and $150 \mathrm{mmHg}$. The respiration target is a partial pressure of carbon dioxide in arterial blood between $35 \mathrm{mmHg}$ and $45 \mathrm{mmHg}$. Patients with respiratory failure requiring $>50 \% \mathrm{O}_{2}$, even with adequate PEEP to maintain sufficient oxygenation, or those experiencing $\mathrm{SpO}_{2}<94 \%$ will be excluded from the trial. Patients will be sedated (propofol and/or midazolam), given analgesia (fentanyl), and paralyzed with neuromuscular blocking agents (rocuronium bromide) during the intervention. Following the completion of the intervention, the ventilator will be changed from the study gas inhalation system to the conventional ventilator available in the ICU. The ventilation mode will be tailored to each patient. Additionally, the neuromuscular blocking agents can be discontinued if they are unnecessary.

\section{Concurrent therapy}

TTM will be performed in all patients. The body temperature will be controlled using surface or intravascular temperature management devices to achieve a target core temperature between $33{ }^{\circ} \mathrm{C}$ and $36{ }^{\circ} \mathrm{C}$. The choice of the target temperature between $33{ }^{\circ} \mathrm{C}$ and $36^{\circ}$ $\mathrm{C}$ is determined by individual institutional policy. However, efforts will be made to achieve the target temperature as soon as possible, and the target temperature will be maintained for $24 \mathrm{~h}$. Patients will be passively rewarmed to a core temperature of $36{ }^{\circ} \mathrm{C}$ over $48 \mathrm{~h}$. Sedation will be stopped upon reaching a temperature of $36{ }^{\circ} \mathrm{C}$ in patients undergoing TTM at $32{ }^{\circ} \mathrm{C}$ to $35^{\circ} \mathrm{C}$. In patients who are managed with TTM at $36{ }^{\circ} \mathrm{C}$, sedation will be stopped $72 \mathrm{~h}$ after initiation of the study gas inhalation. Hyperthermia will be avoided by using icepacks and nonsteroidal anti-inflammatory drugs to maintain a temperature of $37 \pm 0.5{ }^{\circ} \mathrm{C}$ until 7 days post-ROSC for all patients.

Patients will be treated using standard therapies for cardiac diseases. Coronary angiography and percutaneous coronary interventions will be performed according to current guidelines and at the discretion of the treating physicians. Other diagnostic testing will be performed only as indicated after admission. 


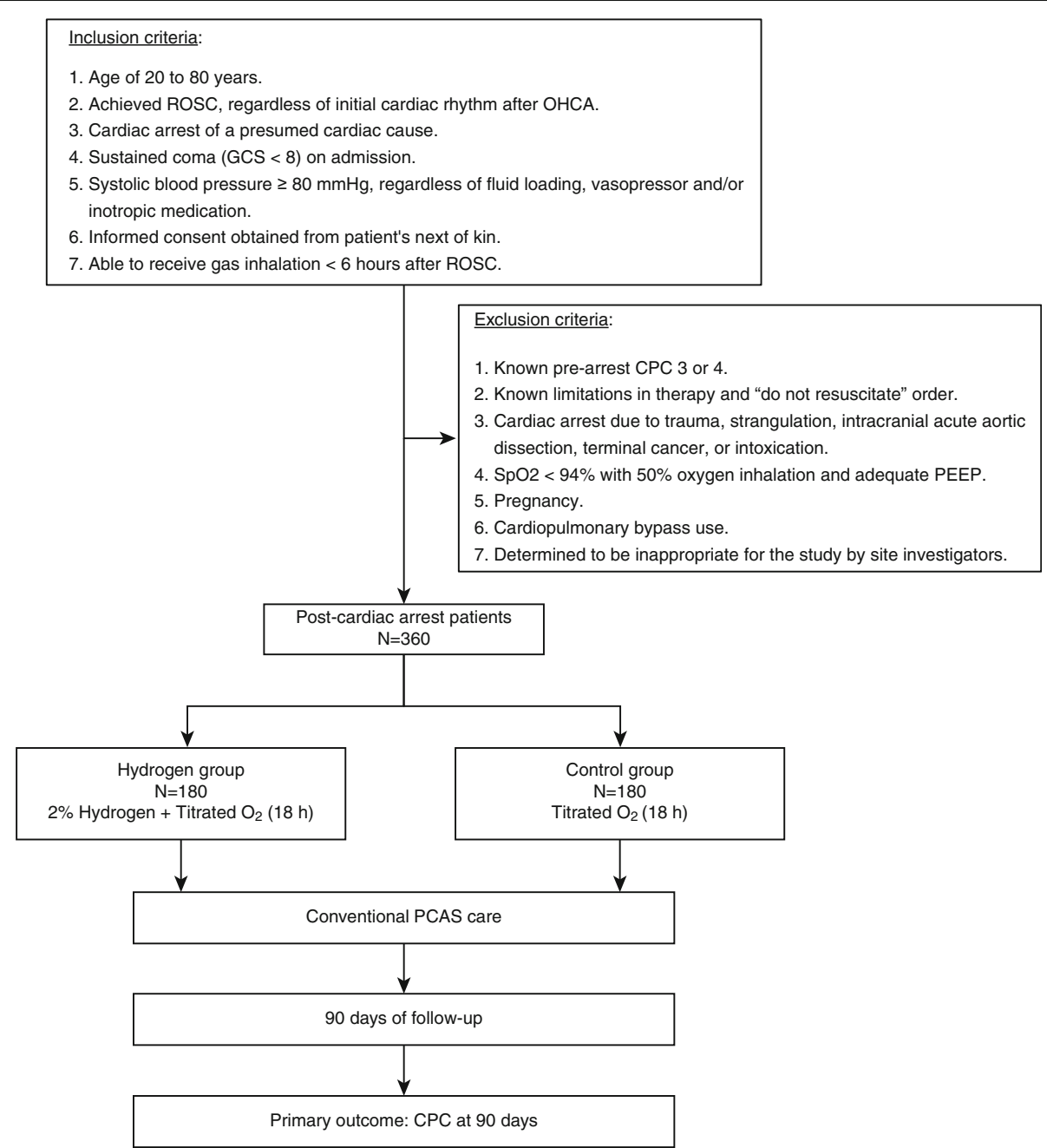

Fig. 1 Flow diagram of the study design. Patients will be randomly allocated 1:1 to the hydrogen group or the control group using contentblinded cylinders. Trial gas inhalation will be initiated after intensive care unit admission and continued for $18 \mathrm{~h}$. Multidisciplinary treatments, including targeted temperature management, will be performed in all patients according to the latest International Liaison Committee on Resuscitation (ILCOR) guidelines. CPC Cerebral Performance Categories scale, GCS Glasgow Coma Scale, PCAS Post-cardiac arrest syndrome, PEEP Positive end-expiratory pressure, $\mathrm{ROSC}$ Return of spontaneous circulation, $\mathrm{SpO}_{2}$ Peripheral oxygen saturation

\section{Data collection and follow-up}

Each participant's baseline demographics and medical history will be collected. All patient data will be collected on a dedicated case report form (CRF). Vital signs, clinical biochemistry, physiological, and radiographic test results will be collected as scheduled (Fig. 3). Patients will be followed until 90 days after CA. Survival status will be recorded until 90 days after CA. The $90-$ day neurological evaluation will be performed by a neurologist or neurosurgeon at each institution and reported through the designated CRFs. Next, two prespecified neurologists outside the study group who are unaware of the treatment will determine the 90-day neurological outcome by evaluating the collected CRFs. Collected CRFs will be locked and stored at the Keio University
School of Medicine. All patients in the study will be actively treated, and brain damage biomarkers will not be used for operational prognostication. All centers will be regularly monitored for source data documentation, and missing or questionable data will be completed and corrected by queries.

\section{Outcome measures}

The primary outcome is the proportion of participants achieving a favorable 90-day neurological outcome as defined by CPCs of 1 and 2 [14]. Secondary outcomes include the 90-day survival rate, survival time, GCS score, modified Rankin Scale (mRS) score, and Mini Mental State Examination (MMSE) results. Cognitive impairment among OHCA survivors is well known, and 


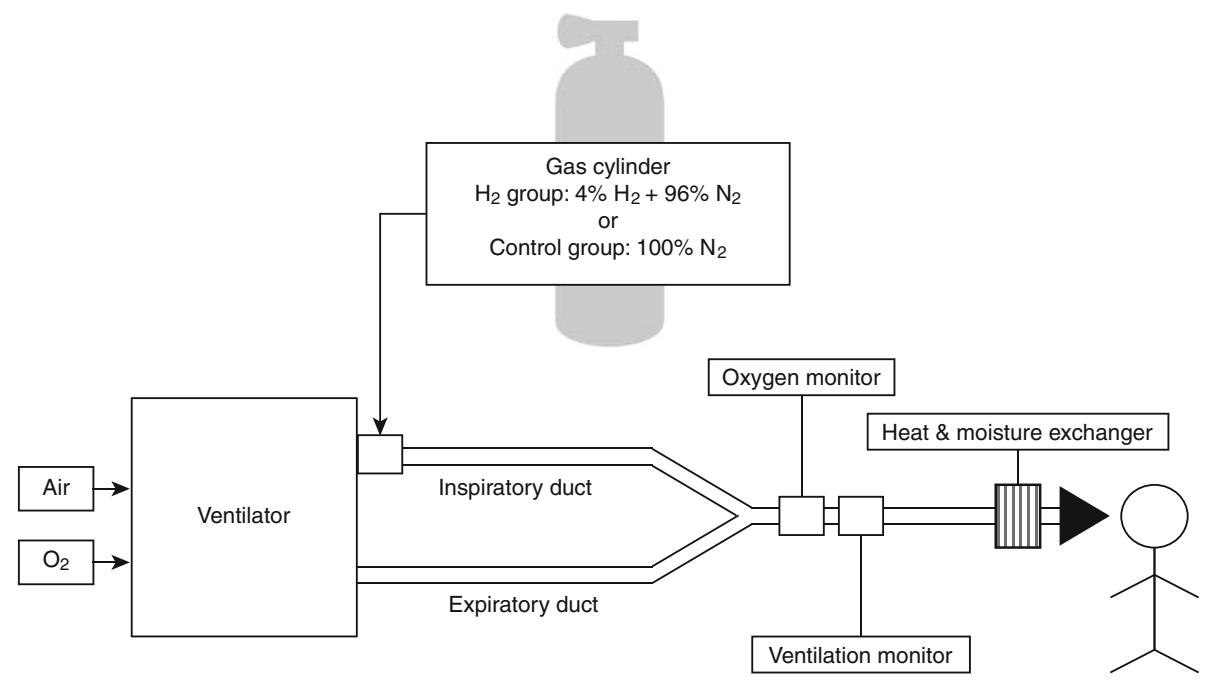

Fig. 2 System of trial gas inhalation. The trial gas will be inhaled through this system. Trial gas will be supplied from a cylinder with constant flow, and it will be mixed with oxygen from the mechanical ventilator (SERVO-s ${ }^{*}$; MAQUET Critical Care AB) at the inspiratory duct. The cylinder is filled with $4 \% \mathrm{H}_{2}$ and $96 \%$ nitrogen for the hydrogen group and 100\% nitrogen for the control group. A tidal volume and fraction of inspired oxygen meter will be used during trial gas inhalation to monitor ventilation

MMSE is the most commonly used instrument for brief cognitive function screening $[15,16]$. We will also collect GCS scores for supplementary evaluation of the prevalence of disturbance of consciousness.

\section{Adverse events}

Patients with PCAS are already in a life-threatening condition following ischemia-reperfusion injury, and various clinical complications, including death, can manifest independent of HI. Therefore, persistent disability/incapacity or a life-threatening condition may not necessarily indicate a serious adverse event (SAE) if it was predictable from the patient's clinical condition. No exceptional rules exist for defining or reporting adverse events (AEs) in studies on patients with PCAS. Therefore, in order to proceed with the study smoothly while fully considering safety, we need to define AEs and establish a reporting rule whereby any newly emergent conditions and clinically significant worsening of the patient's underlying condition, including death, should be recorded in all circumstances. Thus, we defined an AE as any unfavorable medical occurrence that is temporally associated with the participant's involvement in the research, regardless of whether it is considered to be related to participating in the study after HI initiation until the end of the 90day follow-up period. A clinically unfavorable medical occurrence is defined as death, life-threatening worsening of conditions (Additional file 2), permanent or severe organ dysfunction (Additional file 2: Table S1), laboratory abnormalities (Additional file 2: Table S2), seizure, and any other condition that the investigators judge as representing a significant hazard. Considering the above- referenced characteristics of patients with PCAS, an SAE is defined as an AE that occurs within $72 \mathrm{~h}$ after the initiation of the intervention or death during the follow-up period. Safety variables will be recorded in a prespecified CRF.

\section{Sample size estimation}

On the basis of published data [17], we expect the rate of favorable outcome achieved with the current comprehensive critical care, including TTM, in this population to be $50 \%$. Because there are limited clinical data [13], we considered animal data [12] and assumed that the absolute risk reduction by $\mathrm{HI}$ is $15 \%$; that is, the favorable neurological outcome rate improves from $50 \%$ to $65 \%$ with HI. A sample size of 167 patients in each group will provide $80 \%$ power to detect a $15 \%$ change in the proportion of good neurological outcomes (CPCs of 1 and 2), from $50 \%$ to $65 \%$, with an overall significance level of 0.05 according to a two-sided $\chi^{2}$ test. A low rate of failure to follow-up is anticipated because of the short duration of the follow-up and the consideration of recent experiences in our previous study. Including an assumed incomplete patient data rate of $5 \%, 180$ patients are required for each group, for a total sample size of 360 .

\section{Statistical methods}

All statistical analyses, including the interim analysis, will be thoroughly performed by independent statisticians who are not involved in patient treatment or outcome assessment. Statisticians are blinded to the allocation code. Statisticians will perform statistical 


\begin{tabular}{|c|c|c|c|c|c|c|c|c|}
\hline \multirow[b]{3}{*}{ TIMEPOINT } & \multicolumn{8}{|c|}{ STUDY PERIOD } \\
\hline & \multirow{2}{*}{$\begin{array}{c}\text { Enrollment } \\
\text { Less than } 6 \\
\mathrm{~h} \text { from } \\
\text { ROSC }\end{array}$} & \multirow{2}{*}{$\begin{array}{c}\text { Allocation } \\
\text { Less than } \\
6 \mathrm{~h} \text { from } \\
\text { ROSC } \\
0 \mathrm{~h} \\
\end{array}$} & \multicolumn{5}{|c|}{ Post-allocation } & \multirow{2}{*}{$\begin{array}{c}\text { Close-out } \\
90 \mathrm{~d}\end{array}$} \\
\hline & & & $18 \mathrm{~h}$ & $24 \mathrm{~h}$ & $48 \mathrm{~h}$ & $72 \mathrm{~h}$ & $14,30 \mathrm{~d}$ & \\
\hline \multicolumn{9}{|l|}{ ENROLLMENT: } \\
\hline \multirow{4}{*}{$\begin{array}{r}\text { Eligibility screen } \\
\text { Informed consent from } \\
\text { family } \\
\text { Informed consent from } \\
\text { patient } \\
\text { Allocation }\end{array}$} & $x$ & & & & & & & \\
\hline & $\mathrm{X}$ & & & & & & & \\
\hline & $\mathrm{X}$ & & & & & \multicolumn{2}{|c|}{$\begin{array}{l}\text { As soon as } \\
\text { possible }\end{array}$} & \\
\hline & & $\mathrm{x}$ & & & & & & \\
\hline \multicolumn{9}{|l|}{ INTERVENTIONS: } \\
\hline \multicolumn{9}{|l|}{$\left[\mathrm{O}_{2}+2 \%\right.$ hydrogen] } \\
\hline \multicolumn{9}{|l|}{$\left[\mathrm{O}_{2}\right]$} \\
\hline \multicolumn{9}{|l|}{ ASSESSMENTS: } \\
\hline \multirow{4}{*}{$\begin{array}{l}\text { Baseline variables: } \\
\text { Sex, age, comorbidities, } \\
\text { Pre-hospital variables: } \\
\text { Location of arrest, } \\
\text { witnessed arrest, } \\
\text { bystander CPR, initial } \\
\text { documented rhythm, time } \\
\text { to ROSC } \\
\mathrm{HR}, \mathrm{BP}, \mathrm{SpO}_{2}, \mathrm{FiO}_{2} \text {, } \\
\mathrm{PEEP}^{\text {other }} \\
\text { hemodynamic } \\
\text { parameters }\end{array}$} & $x$ & & & & & & & \\
\hline & $x$ & & & & & & & \\
\hline & & & $x$ & $x$ & $X$ & $x$ & & \\
\hline & & & $x$ & $x$ & $\mathrm{X}$ & $x$ & & \\
\hline Concomitant drugs & $\mathrm{x}$ & & & $x$ & $\mathrm{X}$ & $x$ & & \\
\hline \multirow{3}{*}{$\begin{array}{l}\text { EEG } \\
\text { Laboratory test (blood, ur } \\
\text { ine, arterial gas analysis) }\end{array}$} & $x$ & & & & & $X^{*}$ & & \\
\hline & & & & & & $X^{*}$ & & \\
\hline & $x$ & $x$ & $x$ & $x$ & $x$ & $x$ & & \\
\hline \multirow{2}{*}{ AEs } & & & $x$ & $x$ & $\mathrm{x}$ & $x$ & $x$ & $x$ \\
\hline & & & & & & $x$ & $x$ & $x$ \\
\hline \multirow{2}{*}{ mRS, MMSE } & & & & & & & $x$ & $x$ \\
\hline & $x$ & $x$ & $x$ & $x$ & $\mathrm{X}$ & $x$ & $x$ & $x$ \\
\hline
\end{tabular}

Fig. 3 Standard Protocol Items: Recommendations for Interventional Trials (SPIRIT) figure depicting schedule of enrollment, interventions, and assessments. ${ }^{\dagger}$ Hemodynamic parameters will be obtained using commercially available systems (PiCCO ${ }^{\oplus}, M^{2}$ AUET Critical Care AB; or $\mathrm{EV} 1000^{\oplus}$, Edwards Lifesciences, Irvine, CA, USA). * Perform head CT scan and EEG during the daytime after $72 \mathrm{~h}$ from the initiation of intervention. $A B G$ Arterial blood gas analysis, AEs Adverse events, BP Blood pressure, CAG Coronary angiography, CBC Complete blood count, CPC Cerebral Performance Categories scale, CT Computed tomography, ECG Electrocardiogram, EEG Electroencephalogram, ER Emergency room, $\mathrm{FiO}_{2}$ Fraction of inspired oxygen, GCS Glasgow Coma Scale, HR Heart rate, MMSE Mini Mental State Examination, mRS Modified Rankin Scale, $\mathrm{PCI}$ Percutaneous coronary intervention, PEEP Positive end-expiratory pressure, $\mathrm{SpO}_{2}$ Peripheral oxygen saturation

analyses according to predetermined data handling and statistical methods, and there will be no arbitrary interference. All analyses will be performed on a full-analysis basis. Baseline characteristics by group will be compared using descriptive analyses. The proportion of patients achieving good neurological outcomes at the end of the follow-up will be compared using the Pearson $\chi^{2}$ test, which will be the primary result of the trial. With regard to the secondary outcomes, the 90-day survival rate will be compared using the Pearson $x^{2}$ test; the duration of survival will be analyzed using Cox regression analysis; and other neurological outcomes, such as mRS, GCS, 
and MMSE scores, will be compared using the MannWhitney $U$ test. All tests will be two-tailed, and a $P$ value of 0.05 will be considered statistically significant. All data analyses will be performed using SAS version 9.3 software (SAS Institute, Cary, NC, USA).

\section{Interim analysis}

The interim analysis of safety will be conducted by the data and safety monitoring committee (DSMC) to determine whether to continue the study after the first 100 patients have been followed for 90 days. This analysis will be performed only for death (within the 90th day of the study). In the interim analysis, we will make a statistical estimate comparing mortality between the hydrogen and control groups. For each group, an independent beta distribution $[\beta(1,1)]$ will be used as a prior distribution, and the posterior distribution of the mortality rate of each group will be estimated on the basis of number of deaths observed. Based on the aforementioned distribution, the criteria for the discontinuation of the study will be met when the probability that the mortality rate of the hydrogen group is higher than that of the control group is $\geq 0.9875$.

\section{Data and safety monitoring committee}

The DSMC is an independent group consisting of clinicians who have experience in the management of ICU patients and a statistician who has experience in the execution, monitoring, and analysis of clinical trials. DSMC membership is restricted to nonparticipating clinicians without conflicts of interest. The DSMC will be responsible for safeguarding the interests of trial participants, assessing the causal relationship between reported AEs and the intervention, and the interim analysis. The DSMC will provide recommendations regarding stopping or continuing the trial to the principal investigator of the trial.

\section{Auditing}

Auditing will be outsourced to a contract research organization independent of the investigators and the sponsor. Four institutions will be randomly chosen annually, and auditing will be performed according to the predefined procedure.

\section{Discussion}

$\mathrm{HI}$ is a promising treatment option for PCAS. Because PCAS is observed in a unique group of patients and has a serious and urgent nature, it is rarely studied in double-blind RCTs. The HYBRID II trial will be the first randomized double-blind trial to evaluate the efficacy of HI.

Animal experimental data support the benefits of $\mathrm{HI}$ for PCAS [11, 12]. Therapeutic effects of $\mathrm{HI}$ at concentrations ranging from $1.3 \%$ to $3 \%$ have been reported in various models. We have chosen an $\mathrm{H}_{2}$ concentration of $2 \%$ because the protective effect against acute oxidative stress plateaued at over $2 \%$ in previous reports $[8,18]$. Synergistic effects of TTM and HI have been reported in rodent PCAS models [11, 12]. TTM and $\mathrm{HI}$ may share the same theoretical neuroprotective mechanisms. However, other yet to be defined mechanisms are speculated to exist.

Although $\mathrm{H}_{2}$ gas is flammable, concentrations $<4 \%$ together with oxygen at room temperature are incombustible. As indicated by the second law of thermodynamics, although several physical processes that satisfy the first law are possible, the only processes that occur in nature are those for which the entropy of the system either remains constant or increases. Thus, exhaled $\mathrm{H}_{2}$ diffuses instantly, not accumulating or resulting in an increased concentration exceeding that of the inspiratory $\mathrm{H}_{2}$ concentration. Therefore, $2 \% \mathrm{H}_{2}$ gas can be solicitously administered in a hospital.

$\mathrm{HI}$ at a therapeutic dose has been reported to have no adverse effects on vital signs [18]. No unfavorable effects have been reported with the prolonged repetitive inhalation of $\mathrm{H}_{2}$ to prevent decompression sickness in healthy deep-sea divers $[19,20]$.

Comatose post-CA patients generally require mechanical ventilation. Therefore, $\mathrm{H}_{2}$ must be administered through a mechanical ventilator circuit. We have successfully devised a ventilator system that enables simultaneous administration of titrated $\mathrm{H}_{2}$ and $\mathrm{O}_{2}$ (Fig. 2) using a clinically available ventilator. A significant point to emphasize is that platinum is a catalyst for the oxidation reaction of $\mathrm{H}_{2}$. Thus, even if $\mathrm{H}_{2}$ itself is incombustible under $4 \%$ concentration at room temperature, the platinum surface may be overheated in the presence of $\mathrm{H}_{2}$ and $\mathrm{O}_{2}$, eventually leading to mechanical failure. This is essential because most of the current clinically available ventilators are equipped with a platinum hot manometer as a flow sensor, thereby limiting ventilator options for the administration of $\mathrm{H}_{2}$ gas. We chose an existing ventilator equipped with an ultrasonic flow sensor $\left(\right.$ Servo- $\left.\mathrm{s}^{\circ}\right)$. The feasibility and safety of HI using this system was confirmed in a small sample size of subjects with PCAS [13]. A clinical trial evaluating the efficacy of $\mathrm{HI}$ for PCAS is eagerly anticipated [21].

Our HI system has several limitations. First, the maximum $\mathrm{O}_{2}$ concentration is limited to $50 \%$. Although PEEP will be tailored to maintain sufficient oxygenation, patients requiring $>50 \% \mathrm{O}_{2}$ must be excluded. Second, HI is limited to $18 \mathrm{~h}$ because of space limitations for the installation of cylinders in the Japanese ICU. The gas cylinder that will be used in this trial is capable of providing gas for approximately 10 to $12 \mathrm{~h}$, depending on the flow rate. 
Considering the space limitation, two cylinders per patient is the maximum. Thus, the trial gas inhalation duration was set for $18 \mathrm{~h}$. There are no conclusive studies on the optimal duration of $\mathrm{HI}$ after $\mathrm{CA}$, even in animal models; thus, $18 \mathrm{~h}$ might be short for some patients. Third, a time lag between ROSC and $\mathrm{HI}$ initiation is inevitable because the $\mathrm{HI}$ system will need to be installed in the ICU. $\mathrm{H}_{2}$ has been reported to ameliorate ischemia-reperfusion injury in part by radical-scavenging effects; it is easily assumed that earlier initiation of HI after ROSC in the ER may demonstrate better results. However, our results derived from animal experiments revealed that the benefit of HI is similar, even when it is initiated following ROSC [12]. The time delay for obtaining consent is minimal because consent is usually obtained from a proxy while the patient is sent for emergent revascularization. A deferred consent approach was not chosen, because consent from a proxy was mandated by the Japanese government for approval of this trial as an advanced medicine clinical trial. Based on the abovementioned reasons, a time lag $<6 \mathrm{~h}$ between starting $\mathrm{HI}$ after ROSC is considered realistic. According to our previous experience, it is expected that the refusal rate will not be sufficient to produce a selection bias.

We have designed a pragmatic randomized trial with wide inclusion criteria after taking previous trials, clinical guidelines, and current clinical practices into consideration. Wide inclusion criteria will strengthen the generalizability of our results.

In this trial, we will arrange randomization and blinding in a unique way by using ingredient-concealed, prerandomized cylinders. It is practically impossible to stock certain sets of large cylinders at each hospital and choose them according to central computer-based randomization. The blinding and 1:1 allocation will be maintained without confusion by simply using the delivered cylinders in numerical order at each site. Scrupulous trial design, including monitoring and auditing by external contract research organizations, is planned to maintain compliance and eventually to obtain high-quality evidence. Moreover, this trial has been approved as an advanced medicine clinical trial whose aim is to obtain future regulatory approval by the Ministry of Health, Labor and Welfare of the Japanese government.

$\mathrm{H}_{2}$ is one of the most abundant substances in the universe, and it is far less expensive and is effective at lower concentrations than other noble gases that have been studied for PCAS [22]. The unique features of $\mathrm{HI}$ include a less invasive nature, easy and safe administration, and no obvious known adverse effects. Therefore, our expectation is that once $\mathrm{H}_{2}$ is demonstrated to be effective, $\mathrm{HI}$ will have the potential to be widely applied to patients with PCAS.

\section{Trial status and time line}

As of 19 October 2017, 2 patients were enrolled and recruitment is ongoing. Approximately 15 institutions are preparing to begin patient enrollment. A total of $360 \mathrm{pa}-$ tients will be recruited for the trial within 3 years.

\section{Additional files}

Additional file 1: Completed Standard Protocol Items: Recommendations for Interventional Trials (SPIRIT) checklist of recommended items to address in a clinical trial protocol. (DOC $125 \mathrm{~kb}$ )

Additional file 2: Definition of adverse event. (DOCX $26 \mathrm{~kb}$ )

\section{Abbreviations}

ABG: Arterial blood gas analysis; AE: Adverse event; BP: Blood pressure;

CA: Cardiac arrest; CAG: Coronary angiography; CBC: Complete blood count; CPC: Cerebral Performance Categories scale; CRF: Case report form;

$\mathrm{CT}$ : Computed tomography; DSMC: Data and safety monitoring committee; ECG: Electrocardiogram; EEG: Electroencephalogram; ER: Emergency room; $\mathrm{FiO}_{2}$ : Fraction of inspired oxygen; GCS: Glasgow Coma Scale; $\mathrm{H}_{2}$ : Molecular hydrogen; HI: Hydrogen gas inhalation; HR: Heart rate; HYBRID Il: Efficacy of inhaled HYdrogen on neurological outcome following BRain Ischemia During post-cardiac arrest care; ICU: Intensive care unit; ILCOR: International Liaison Committee on Resuscitation; MMSE: Mini Mental State Examination; mRS: Modified Rankin Scale; OHCA: Out-of-hospital cardiac arrest; PCAS: Postcardiac arrest syndrome; PCl: Percutaneous coronary intervention; PEEP: Positive end-expiratory pressure; RCT: Randomized, double-blind, placebo-controlled trial; ROSC: Return of spontaneous circulation; SAE: Serious adverse event; SPIRIT: Standard Protocol Items: Recommendations for Interventional Trials; $\mathrm{SpO}_{2}$ : Peripheral oxygen saturation; TTM: Targeted temperature management; UMIN: University Hospital Medical Information Network

\section{Acknowledgements}

The authors thank Dr. N. Kamatani for providing the statistical analysis design and assisting with preparation of the manuscript.

\section{Funding}

This trial will be funded by the Taiyo Nippon Sanso Corporation, including the provision of the gas (hydrogen or nitrogen) and the cost for cylinder delivery. Ordinary post-CA care, including TTM, will be insured by the Japanese health system. The funder of this study will not be involved in study design; data collection, management, analysis, or interpretation; the writing of the report; or the decision to submit the manuscript for publication.

\section{Availability of data and materials}

All de-identified individual participant data, study protocols, statistical analysis plans, and analytic codes will be shared with investigating members of the HYBRID Study Group. Shared data may be used for any type of analysis. Data will be available from the HYBRID Study Office at Keio University for 5 years immediately following publication.

\section{Authors' contributions}

TT, MSu, MSa, SO, and $\mathrm{KH}$ designed the study. TT wrote the manuscript. MSu, and MSa critically reviewed the study proposal and approved the final version to be published. All authors read and approved the final manuscript.

\section{Ethics approval and consent to participate}

This protocol was approved by the ethics committee of the Keio University School of Medicine (reference number 20150266). Additionally, this trial has been approved as an advanced medicine clinical trial by the Ministry of Health, Labor and Welfare of the Japanese government (reference number 1117-2). Ethics committee approval will be obtained at each participating hospital, and recruitment of patients will not start in those hospitals unless this study is approved by the ethics committee of that hospital. All amendments will be submitted for approval of the ethics committee of the Keio University School of Medicine and the Ministry of Health, Labor and Welfare of the Japanese government. A 
detailed description of obtaining consent to participate is provided within the text of this article.

\section{Consent for publication}

Not applicable.

\section{Competing interests}

The authors declare that they have no competing interests.

\section{Publisher's Note}

Springer Nature remains neutral with regard to jurisdictional claims in published maps and institutional affiliations.

\section{Author details}

'Department of Emergency and Critical Care Medicine, Keio University School of Medicine, 35 Shinanomachi, Shinkuku-ku, Tokyo 160-8582, Japan.

${ }^{2}$ The Center for Molecular Hydrogen Medicine, Keio University, 2-15-45 Mita, Minato-ku, Tokyo 108-8345, Japan. ${ }^{3}$ Department of Cardiology, Keio University School of Medicine, 35 Shinanomachi, Shinkuku-ku, Tokyo 160-8582, Japan

\section{Received: 21 February 2017 Accepted: 5 October 2017}

Published online: 23 October 2017

\section{References}

1. Moulaert VR, Verbunt JA, van Heugten CM, Wade DT. Cognitive impairments in survivors of out-of-hospital cardiac arrest: a systematic review. Resuscitation. 2009:80:297-305. doi:10.1016/j.resuscitation.2008.10.034

2. Arrich J, Holzer M, Havel C, Mullner M, Herkner H. Hypothermia for neuroprotection in adults after cardiopulmonary resuscitation. Cochrane Database Syst Rev. 2016;2:CD004128. doi:10.1002/14651858.CD004128.pub4.

3. Peberdy MA, Callaway CW, Neumar RW, Geocadin RG, Zimmerman JL, Donnino M, et al. Part 9: post-cardiac arrest care: 2010 American Heart Association Guidelines for Cardiopulmonary Resuscitation and Emergency Cardiovascular Care. Circulation. 2010;122(18 Suppl 3):S768-86. doi:10.1161/ CIRCULATIONAHA.110.971002. Published errata appear in Circulation. 2011; 123:e237 and Circulation. 2011;124:e403.

4. Callaway CW, Donnino MW, Fink EL, Geocadin RG, Golan E, Kern KB, et al. Part 8: post-cardiac arrest care: 2015 American Heart Association Guidelines Update for Cardiopulmonary Resuscitation and Emergency Cardiovascular Care. Circulation. 2015;132(18 Suppl 2):S465-82. doi:10.1161/CIR. 0000000000000262.

5. Cariou A, Payen JF, Asehnoune K, Audibert G, Botte A, Brissaud O, et al. Targeted temperature management in the ICU: guidelines from a French expert panel. Ann Intensive Care. 2017;7:70. doi:10.1186/s13613-017-0294-1.

6. De Keyser J, Sulter G, Luiten PG. Clinical trials with neuroprotective drugs in acute ischaemic stroke: are we doing the right thing? Trends Neurosci. 1999:22:535-40.

7. Huang L, Applegate PM, Gatling JW, Mangus DB, Zhang J, Applegate 2nd RL. A systematic review of neuroprotective strategies after cardiac arrest: from bench to bedside (part II-comprehensive protection). Med Gas Res. 2014:4:10. doi:10.1186/2045-9912-4-10.

8. Ohsawa I, Ishikawa M, Takahashi K, Watanabe M, Nishimaki K, Yamagata K, et al. Hydrogen acts as a therapeutic antioxidant by selectively reducing cytotoxic oxygen radicals. Nat Med. 2007;13:688-94. doi:10.1038/nm1577.

9. Juchi K, Imoto A, Kamimura N, Nishimaki K, Ichimiya H, Yokota T, et al. Molecular hydrogen regulates gene expression by modifying the free radical chain reaction-dependent generation of oxidized phospholipid mediators. Sci Rep. 2016;6:18971. doi:10.1038/srep18971.

10. Ichihara M, Sobue S, Ito M, Ito M, Hirayama M, Ohno K. Beneficial biological effects and the underlying mechanisms of molecular hydrogen comprehensive review of 321 original articles. Med Gas Res. 2015;5:12. doi: 10.1186/s13618-015-0035-1.

11. Hayashida K, Sano M, Kamimura N, Yokota T, Suzuki M, Maekawa Y, et al. $H_{2}$ gas improves functional outcome after cardiac arrest to an extent comparable to therapeutic hypothermia in a rat model. J Am Heart Assoc. 2012:1:e003459. doi:10.1161/JAHA.112.003459.

12. Hayashida K, Sano M, Kamimura N, Yokota T, Suzuki M, Ohta S, et al Hydrogen inhalation during normoxic resuscitation improves neurological outcome in a rat model of cardiac arrest independently of targeted temperature management. Circulation. 2014;130:2173-80. doi:10.1161/ CIRCULATIONAHA.114.011848.

13. Tamura T, Hayashida K, Sano M, Suzuki M, Shibusawa T, Yoshizawa J, et al. Feasibility and safety of hydrogen gas inhalation for post-cardiac arrest syndrome - first-in-human pilot study. Circ J. 2016;80:1870-3. doi:10.1253/ circj.CJ-16-0127.

14. Becker LB, Aufderheide TP, Geocadin RG, Callaway CW, Lazar RM, Donnino MW, et al. Primary outcomes for resuscitation science studies: a consensus statement from the American Heart Association. Circulation. 2011;124:215877. doi:10.1161/CIR.0b013e3182340239.

15. Green CR, Botha JA, Tiruvoipati R. Cognitive function, quality of life and mental health in survivors of our-of-hospital cardiac arrest: a review. Anaesth Intensive Care. 2015:43:568-76.

16. Nichol G, Guffey D, Stiell IG, Leroux B, Cheskes S, Idris A, et al. Postdischarge outcomes after resuscitation from out-of-hospital cardiac arrest: a ROC PRIMED substudy. Resuscitation. 2015;93:74-81. doi:10.1016/j. resuscitation.2015.05.011.

17. Nielsen N, Wetterslev J, Cronberg T, Erlinge D, Gasche $Y$, Hassager C, et al. Targeted temperature management at $33^{\circ} \mathrm{C}$ versus $36^{\circ} \mathrm{C}$ after cardiac arrest. N Engl J Med. 2013;369:2197-206. doi:10.1056/NEJMoa1310519.

18. Hayashida K, Sano M, Ohsawa I, Shinmura K, Tamaki K, Kimura K, et al. Inhalation of hydrogen gas reduces infarct size in the rat model of myocardial ischemia-reperfusion injury. Biochem Biophys Res Commun. 2008:373:30-5. doi:10.1016/j.bbrc.2008.05.165.

19. Lafay V, Barthelemy P, Comet B, Frances Y, Jammes Y. ECG changes during the experimental human dive HYDRA 10 (71 atm/7,200 kPa). Undersea Hyperb Med. 1995;22:51-60.

20. Fontanari P, Badier M, Guillot C, Tomei C, Burnet H, Gardette B, et al. Changes in maximal performance of inspiratory and skeletal muscles during and after the 7.1-MPa Hydra 10 record human dive. Eur J Appl Physiol. 2000;81:325-8

21. Drabek T, Kochanek PM. Improving outcomes from resuscitation: from hypertension and hemodilution to therapeutic hypothermia to $\mathrm{H}_{2}$. Circulation. 2014;130:2133-5. doi:10.1161/CIRCULATIONAHA.114.013566.

22. Laitio R, Hynninen M, Arola O, Virtanen S, Parkkola R, Saunavaara J, et al. Effect of inhaled xenon on cerebral white matter damage in comatose survivors of out-of-hospital cardiac arrest: a randomized clinical trial. JAMA 2016;315:1120-8. doi:10.1001/jama.2016.1933.

\section{Submit your next manuscript to BioMed Central and we will help you at every step:}

- We accept pre-submission inquiries

- Our selector tool helps you to find the most relevant journal

- We provide round the clock customer support

- Convenient online submission

- Thorough peer review

- Inclusion in PubMed and all major indexing services

- Maximum visibility for your research

Submit your manuscript at www.biomedcentral.com/submit
Biomed Central 